\title{
THE
}

\section{Comparison of diet quality, physical activity and biochemical values of older adults either reporting or not reporting use of lipid- lowering medication}

Ingrid E. Lofgren

University of Rhode Island, ingrid_lofgren@uri.edu

Geoffrey Greene

University of Rhode Island, ggreene@uri.edu

S. Schembre

University of Rhode Island

Matthew J. Delmonico

University of Rhode Island, delmonico@uri.edu

Deborah Riebe

University of Rhode Island, debriebe@uri.edu

Follow this and additional works at: https://digitalcommons.uri.edu/nfs_facpubs See next page for additional authors

This is a pre-publication author manuscript of the final, published article.

Terms of Use

All rights reserved under copyright.

\section{Citation/Publisher Attribution}

Lofgren, I., Greene, G., Schembre, S. et al. J Nutr Health Aging (2010) 14: 168. https://doi.org/10.1007/ s12603-010-0030-0

Available at: https://doi.org/10.1007/s12603-010-0030-0

This Article is brought to you for free and open access by the Nutrition and Food Sciences at DigitalCommons@URI. It has been accepted for inclusion in Nutrition and Food Sciences Faculty Publications by an authorized administrator of DigitalCommons@URI. For more information, please contact digitalcommonsgroup@uri.edu. 


\section{Authors}

Ingrid E. Lofgren, Geoffrey Greene, S. Schembre, Matthew J. Delmonico, Deborah Riebe, and P. Clark 


\title{
Comparison of Diet Quality, Physical Activity and Biochemical Values of Older Adults either Reporting or Not Reporting Use of Lipid-Lowering Medication
}

\author{
Ingrid Lofgren ${ }^{\mathrm{a}}$, Geoffrey Greene ${ }^{\mathrm{a}}$, Susan Schembre ${ }^{\mathrm{a}}$, Matthew J. Delmonico ${ }^{\mathrm{b}}$, Deb Riebe ${ }^{\mathrm{b}}$, \\ and Phillip Clark ${ }^{\mathrm{C}}$ \\ aDepartment of Nutrition and Food Sciences, University of Rhode Island, Ranger Hall, Kingston, \\ Rhode Island, 02881, United States \\ bDepartment of Kinesiology, University of Rhode Island, Independence Square II, Rhode Island, \\ 02881, United States \\ 'Program in Gerontology, University of Rhode Island, Quinn Hall, Rhode Island, 02881, United \\ States
}

\section{Abstract}

Objectives-The aim of this study was to compare standard lipid profile, reported dietary intake, and physical activity in older adults who reported taking or not taking a lipid-lowering medication, namely statins.

Design-Cross-sectional study utilizing baseline data collected from a subsample of a larger randomized clinical trial, The Study of Exercise and Nutrition in Older Rhode Islanders (SENIOR) Project.

Participants and Setting-A total of 115 participants, 33 males and 82 females, over the age of 60, community-dwelling, primarily retired, and from East Providence, Rhode Island and surrounding communities in Rhode Island and Massachusetts.

Measurements-Height and weight were measured and used to calculate body mass index. Waist circumference was measured. Medical history and medication use surveys were completed. Dietary assessment was done via three 24 hour recalls using NDS-R. The Yale Physical Activity Survey was used to determine energy expenditure during exercise and a physical activity summary score. Fasting blood samples were obtained to determine lipid profile.

Results-37 participants (32.2\%) reported taking lipid-lowering medication, statins exclusively, and $78(67.8 \%)$ reported not taking any lipid-lowering medication. Participants who reported taking statins had better lipid profiles than those participants who reported not taking statins but had significantly lower intakes of vitamin $B_{12}$, vitamin $K$, calcium, and potassium. There were no differences between groups on reported physical activity. However, the mean intakes for both groups did not meet the Dietary Reference Intakes for multiple nutrients.

Conclusion-Older adults need additional education on the importance of lifestyle changes in reducing CHD risk, whether taking lipid-lowering medications or not.

\section{Keywords}

elderly; statins; dietary intake; cholesterol 


\section{Introduction}

Over $80 \%$ of those who die from coronary heart disease (CHD) are 65 years or older and for those who survive a heart attack, their life expectancy can decrease up to fifteen years (1). Strategies to reduce risk and treat CHD include diet and physical activity modifications, lipid-lowering medications, such as statins, and in some circumstances, surgery (2). The National Heart, Lung, and Blood Institute (NHLBI) and the American Heart Association (AHA) consider lifestyle improvements the first therapeutic option $(2,3)$ and if medications and/or surgery are needed, healthy diets and regular physical activity are critical to maintain the lowest risk profile (2-5). Although many older adults believe diet can manage CHDrelated dyslipidemia, they do not attempt lifestyle modifications in conjunction with medication because it's thought taking statins is easier (6). The lack of lifestyle improvements may be due to an expectation that medications replace healthy lifestyle choices and this belief can result in poor dietary quality and a sedentary lifestyle, which attenuates the effectiveness of lipid-lowering medications (6). Tailored education regarding the synergistic effect of multiple strategies to reduce CHD risk is needed if taking a lipidlowering medication leads to poor lifestyle choices.

Agencies including NHLBI and AHA have similar dietary recommendations for CHD prevention and treatment $(2,7-9) ; 50-60 \%$ carbohydrate, $15 \%$ protein, and $25 \%$ to $35 \%$ total fat ( $<7 \%$ saturated and $\_2 \%$ monounsaturated fat) and $<300 \mathrm{mg}$ of cholesterol. Additional recommendations include 4.5 cups of fruits and vegetables a day and half of grains consumed should be whole grains $(2,9)$. The American College of Sports Medicine (ACSM) recommends that older adults utilize physical activity as a key management tool to prevent and treat CHD and CHD-related disability (10). ACSM recommendations include at least 30 minutes of moderate- intensity aerobic activity five days/week or vigorous activity for at least 20 minutes three days/week (10). Muscle-strengthening and flexibility are each recommended at least two days/week as well as balance activities (10). Older adults with clinically significant CHD may need a supervised activity program. Patients at risk for CHD should attempt dietary and physical activity modifications for at least three months prior to starting lipid-lowering medications as some individuals can maintain target lipid levels through these methods exclusively (2). If target lipid concentrations cannot be maintained by diet and activity alone, medications and lifestyle modifications can have an additive effect on reducing CHD risk compared to statins alone.

Older adults should rely on multiple risk reduction strategies to prevent and treat CHD for several reasons. Many cannot reach and/or obtain optimal low density lipoprotein cholesterol (LDL-C) concentrations $(<100 \mathrm{mg} / \mathrm{dl})$ on statins alone. Also, prescription compliance is low which reduces the medication's effectiveness. As many as $50 \%$ discontinue statins, the most popular lipid-lowering medication, after one year (11) with rates increasing to 75\% after three years (12). Reasons for poor adherence include cost (13), low expectation of impact, and depression (14). Additionally, negative side effects from statins include abnormal liver enzyme activity and muscle pain (3).

The purpose of this study was to compare lipid biomarkers as well as reported dietary and physical activity behaviors in reported users and non-users of statins in a population of older adults. It is hypothesized that participants taking statins will have a significantly better lipid profile but a less nutrient dense diet and less physical activity than non-statin users. Examining lifestyle choices made by older adults taking and not taking statins could result in tailored CHD risk management education for older adults. 


\section{Methods and Materials}

\section{The SENIOR Project}

This cross-sectional study utilized baseline data collected from a subsample of larger randomized clinical trial, The Study of Exercise and Nutrition in Older Rhode Islanders (SENIOR) Project (15). The SENIOR Project was an intervention study for communitydwelling older adults designed to increase fruit and vegetable intake and physical activity $(15,16)$. The SENIOR Project participants $(\mathrm{N}=1,277)$ were mostly female $(70 \%)$, retired (87\%), and all were over the age of 60. Participants lived in Rhode Island or neighboring communities in Massachusetts at the time of recruitment and had to speak English or Portuguese. Participants were recruited on a rolling basis over a 12 month period via advertisements in the media, flyers, posters and information tables at local supermarkets (17).

\section{Subjects}

Of the 1,277 SENIOR Project participants, a total of 188 male and female participants were recruited for this substudy of nutrition and biomarkers of dietary intake. In addition to the exclusion criteria for the SENIOR Project, 73 participants of the 188 were excluded for having incomplete blood lipid information and/or completed less than two of three valid dietary recalls. Dietary recalls were considered invalid if the interviewer determined, and a supervisor confirmed, that the participant appeared confused about the previous day's dietary intake or reported illness significantly interfering with food intake (18). The resulting sample size for this study was $n=115$. This study was approved by the University of Rhode Island's Institutional Review Board and all participants signed informed consents.

\section{Protocol}

Participant demographic data were collected by SENIOR Project staff via in-person interviews. Approximately one third of SENIOR project participants were asked if they wanted to participate in additional research. All seniors who reported willingness to participate and who were able to be reached by telephone were interviewed by telephone about about medication usage and any medical problems. Two questions that addressed lipid-lowering medication use were asked: "Do you currently take medication to lower your blood cholesterol" and "Do you take a statin drug such as Lipitor, Mevacor, Pravachol, Zocor, Lescol, Baycol or Advicor (Atorvastatin, Lovastatin, Pravastatin, Simvastatin, Fluvastatin, Cerivastatin or Lovastatin plus Niaspan)?" After the phone call, an appointment was scheduled to have anthropometric and biochemical assessments performed at a community hospital. All participants were provided with a food portion visual poster by mail. In the week(s) prior to their assessment appointment, trained staff contacted the participants by phone to perform three 24 hour food recalls. Study staff measured height on a wall-mounted stadiometer to the nearest $1 \mathrm{~cm}$ and a calibrated balance beam scale to the nearest 0.25 pound. Body mass index (BMI), $\mathrm{kg} / \mathrm{m}^{2}$, was then calculated.

\section{Dietary Assessment}

Three 24 hour food recalls were performed between the initial telephone call and scheduled appointment on non-consecutive days; two week days and 1 weekend day. Study staff utilized the multiple pass method when completing the calls, and used the Nutrient Database System for Research (NDS-R) developed by the University of Minnesota (version 4.03_31, 1998-2000, Nutrition Coordinating Center, University of Minnesota, Minneapolis). All records were checked for outliers and missing foods were entered by a supervisor trained on the NDS-R system. The two to three dietary recall results (i.e. kcalories, macronutrient, micronutrients, food groups) per participant were averaged before the analysis took place. 
Pyramid servings of fruits and vegetable intakes from the 24HR were estimated using Pyramid serving data from USDA's CSFII 1994-96 survey database (19). Foods reported in the 24HR, coded using NDS-R, linked to identical or similar food codes in the USDA database. The number of Pyramid servings was calculated as the weight of a food consumed in grams/the weight of a single Pyramid serving of that same food in grams (20).

\section{Physical Activity Assessment}

The Yale Physical Activity Survey (YPAS), an interviewer-administered questionnaire, was used to assess physical activity (21). It has been shown to accurately assess physical activity in older adults as it focuses on the types of activities reported by this specific age group (2224). The YPAS is a comprehensive interviewer-administered survey specifically designed to measure physical activity in older adults. Subjects are asked to estimate time spent in a list of 25 activities, ranging from low- to high-intensity, in a typical week during the last month. Time spent in each activity is multiplied by an intensity code $\left(\mathrm{kcal} \cdot \mathrm{min}^{-1}\right)$ and then summed across all activities to create an index of weekly energy expenditure $\left(\mathrm{kcal} \cdot \mathrm{week}^{-1}\right)$. Subjects are also asked to estimate participation in five activity dimensions: vigorous activity, leisurely walking, moving on feet, standing and sitting. Weights are assigned to each category. The frequency and duration scores are multiplied together and then multiplied again by each dimension's weighing factor to calculate an index for each dimension. The five individual indices are summed to calculate the summary index.

\section{Biochemical Assessment}

Blood samples were obtained with participants in the fasting state ( 12 hours). Blood was collected from an antecubital vein and then centrifuged at $1500 \mathrm{~g}$ in a refrigerated centrifuge for fifteen minutes. Samples were stored in a $-80^{\circ}$ freezer until analysis. Serum was analyzed for TC, high density lipoprotein cholesterol (HDL-C), and TAG in the Miriam Hospital Lipid Research Laboratory. The laboratory participated in the national survey for clinical laboratories sponsored by the College of American Pathologists and the CDC Lipid Standardization Program since 1977. Total cholesterol, HDL-C, and TAG are determined by enzymatic methods on a Beckman CX4 using Beckman reagents. The precipitation method of Gidez et al is used to determine HDL-C (25). The LDL-C concentration is calculated by the Friedewald equation (26) when triglycerides are less than $400 \mathrm{mg} / \mathrm{dl}$. The coefficients of variation for total cholesterol, triglycerides, and HDL-C were $0.8,1.5$, and $2.0 \%$, respectively.

\section{Statistical Analysis}

Data were analyzed via the Statistical Package for Social Sciences (version 16.0, 2007, SPSS Inc, Chicago, IL). Descriptive statistics were performed to ensure normal distribution of the outcome data prior to analyses. For the analysis, fruit and vegetable intake was normalized by square-root transformation (27) and TAG was log transformed. However, the data are shown in untransformed format to facilitate interpretation. Independent sample ttests were performed to determine if there were any mean differences in the biochemical and dietary intake values by group of reported medication use; taking lipid-lowering medications and not taking lipid-lowering medications.

\section{Results}

A total of 115 (33 males and 82 females) participants had complete blood lipid and dietary assessment data. Of this population, 106 (31 males and 75 females) had complete physical activity data. The mean age was $75.2 \pm 6.8$ years, a majority of the sample was Caucasian (82.8\%) and 56\% had completed some high school. Fifty-two percent reported being married or living with someone and $48 \%$ reported being single, widowed, or divorced. Just 
over $86 \%$ were retired, $12.1 \%$ worked part-time, full-time or as homemakers and $1 \%$ were unemployed. Eighty-three percent reported earning less than $\$ 40,000$ a year, $11 \%$ reported $\$ 40,000$ to $\$ 59,999$, and only $6 \%$ reported earning more than $\$ 60,000$ a year. Thirty-seven participants $(32.2 \%)$ reported taking a lipid-lowering medication, statins exclusively and 78 (67.8\%) reported not taking any lipid-lowering medication. A summary of biochemical values is reported in Table 1. As expected, participants who reported taking statins had significantly lower concentrations of total cholesterol (TC) and LDL-C than those participants who reported not taking statins. Triacylglycerol (TAG) and high density lipoprotein cholesterol (HDL-C) concentrations were similar for both groups. There were no differences between groups for the YPAS energy expenditure for exercise score or the YPAS summary score.

A summary of dietary intake of the two groups is reported in Table 2. Caloric intake and macronutrient composition did not vary significantly between the groups. Mean cholesterol intakes were within recommended levels $(<300 \mathrm{mg})$, but mean intake of monounsaturated fats were less than recommendations (up to 20\%) and more than recommended for saturated fat intake (>7\%). Participants not taking lipid-lowering medication reported eating significantly more fruits and vegetables. Participants not taking statins had significantly higher intakes of vitamin $\mathrm{B}_{12}$, vitamin $\mathrm{K}$, calcium, and potassium. However, the mean intakes for both groups did not meet the Dietary Reference Intakes (DRI) for fiber, folate, vitamins $\mathrm{A}, \mathrm{D}, \mathrm{E}$, and $\mathrm{K}$, calcium, magnesium, and potassium and sodium.

\section{Discussion}

Previous research has shown that those who are adherent to lipid-lowering medications make poor dietary choices and are more sedentary because of a feeling of freedom or "permission" and these behaviors do not promote the lowest CHD risk profile $(6,28)$. Lipidlowering medications, especially statins, can have an immediate impact on abnormal lipid profiles and some patients may feel that no other interventions are needed. This is in spite of research showing that diet and exercise can complement statins' effect and the fact that many patients are not compliant with their statin regime. As expected, participants in this study who self-reported statin use had significantly lower TC and LDL-C compared to nonusers. However, statin users also reported significantly lower fruit and vegetable intake as well as lower intakes of vitamin $\mathrm{K}, \mathrm{B}_{12}$, calcium and potassium suggesting a deleterious effect of statin use on dietary quality. Nevertheless, neither group met Dietary Reference Intake (DRI) recommendations for monounsaturated fat, fiber, vitamins A, D, E, K, folate, calcium, magnesium, potassium, and sodium. Contrary to expectations, both groups reported similar weekly physical activity calorie expenditure and amount of time spent exercising. Results suggest that health care professionals should emphasize the importance of healthful dietary choices in addition to medication use with their patients in order to maintain the lowest CHD risk for each individual (29).

Research has shown as few as 50-60\% of participants with elevated TC attempt to make dietary changes prior to starting a lipid-lowering medication $(6,28)$ perhaps because many think taking statins will be easier (6). In one study, only 28 of the 62 (45\%) participants who completed a six month study, were adherent to their statin regime and they were consuming significantly more calories and less fiber than those not adherent to their statin regime. Similarly in the current study, participants who reported taking statins had significantly lower vitamin $\mathrm{K}, \mathrm{B}_{12}$, calcium, potassium and fruit and vegetable intake compared to those reporting no statin use. The poorer diet of those taking statins suggests that older adults taking medication feel as free to avoid healthful dietary choices as previously reported for younger adults. Though the participants who reported not taking statins had better diets relative to statin users, both groups consumed excessive saturated fat, 9.4\%, and lower than 
recommended monounsaturated fat, $11.4 \%$ ( $<7 \%$ and 20\%, respectively). Both groups were also consuming inadequate amounts of fiber, folate, vitamins $\mathrm{A}, \mathrm{D}, \mathrm{E}$, and $\mathrm{K}$, calcium, magnesium, and potassium and more sodium than recommended.

Physical activity is another modifiable risk that can decrease CHD risk. In this study, it was hypothesized that those participants who reported taking statins would report less physical activity than those taking not taking statins; the statins giving them permission to exercise less. However, there was no difference between the two groups in either the YPAS energy expenditure due to exercise or YPAS summary score (Table 1). The energy expenditure for physical activity for the whole group in the current study, 8,378 $\pm 5,379 \mathrm{kcal} / \mathrm{week}$, is similar to the energy expenditure reported by participants attending a community center, $8,125 \pm 4,125 \mathrm{kcal} /$ week (22). The summary score for these participants $(44.7 \pm 24.9$ hours/ month), is similar to 28 male and female participants who had a mean summary index of $41.9 \pm 4.4$ at week 1 and $49.3 \pm 3.7$ at week 14 (24). However, other studies have reported lower energy expenditure for physical activity and higher summary score from the YPAS than this study. In order to determine if the calculated YPAS scores indicate if participants are meeting physical activity recommendations, more research is needed to determine how the levels of energy expended and summary scores correlate with recommendations of time doing exercise and intensity.

One limitation of the study is the use of 24 hour dietary recalls. These recalls can be challenging for older adults as they have a harder time remembering specifics of food intake. The lack of significant dietary findings may have been due to inaccurate dietary records. Though minorities were underrepresented, the distribution of ethnicities was representative of the study's target population (17). Participants were community-dwelling and not in nursing homes or other institutions so generalizability is limited to specific populations of free-living adults. Further, self-reported medication use is not always reliable. The TC and LDL-C concentrations were significantly lower in those taking lipid-lowering medications compared to those not taking lipid-lowering medications that would be expected with statin use suggesting that self-reported statin use was valid.

The results show that though both groups consume less than the recommended levels of multiple nutrients, those who reported taking statins consumed significantly less vitamin K, $\mathrm{B}_{12}$, calcium, potassium, fruits and vegetables than those not taking statins. The poor quality of the diet, as evidenced by these low intakes, suggest that older adults, regardless of their medication regime, needs additional education on improving their dietary intake. The lack of difference between groups on reported physical activity need to be studied in larger, more diverse populations to see if the use of lipid-lowering medications impacts a person's choice to be physically active or not. In addition, research that can provide estimates of what YPAS scores are in relation to ASCM recommendations are needed as well. The results underscore the importance of patient education on utilizing multiple strategies to manage CHD risk even when taking medications that can dramatically reduce CHD risk (30). Though statins may be viewed as the "easier" choice, the combination of lipid-lowering medication with lifestyle changes is highly recommended for all population to further reduce and maintain a low CHD risk.

\section{Acknowledgments}

Supported by the 1RO1AG16588 from the National Institute on Aging, National Institutes of Health with additional National Cancer Institute and Office of Dietary Supplements funding (RFA OD-93-002) and additional NCI supplemental funding for R0IAG16588.

We wish to thank the National Institutes of Health Office of Dietary Supplements, the SENIOR Program staff, and the SENIOR Project participants. 


\section{References}

1. American Heart Association. Heart Disease and Stroke Statistics - 2008 Update. Dallas, Texas: American Heart Association; 2008.

2. Executive Summary of The Third Report of The National Cholesterol Education Program (NCEP) Expert Panel on Detection, Evaluation And Treatment of High Blood Cholesterol In Adults (Adult Treatment Panel III). Jama. 2001; 285:2486-97. [PubMed: 11368702]

3. Jenkins DJ, Kendall CW, Marchie A, et al. Direct comparison of a dietary portfolio of cholesterollowering foods with a statin in hypercholesterolemic participants. Am J Clin Nutr. 2005; 81:380-7. [PubMed: 15699225]

4. Third Report of the National Cholesterol Education Program (NCEP) Expert Panel on Detection, Evaluation, and Treatment of High Blood Cholesterol in Adults (Adult Treatment Panel III) final report. Circulation. 2002; 106:3143-421. [PubMed: 12485966]

5. Grundy SM, Cleeman JI, Merz CN, et al. Implications of recent clinical trials for the National Cholesterol Education Program Adult Treatment Panel III guidelines. Circulation. 2004; 110:22739. [PubMed: 15249516]

6. Mann DM, Allegrante JP, Natarajan S, Montori VM, Halm EA, Charlson M. Dietary indiscretion and statin use. Mayo Clin Proc. 2007; 82:951-7. [PubMed: 17673064]

7. Lichtenstein AH, Appel LJ, Brands M, et al. Diet and lifestyle recommendations revision 2006: a scientific statement from the American Heart Association Nutrition Committee. Circulation. 2006; 114:82-96. [PubMed: 16785338]

8. Seagle HM, Strain GW, Makris A, Reeves RS. Position of the American Dietetic Association: weight management. J Am Diet Assoc. 2009; 109:330-46. [PubMed: 19244669]

9. Nutrition and Your Health: Dietary Guidelines for Americans, 2005. 6. Washington, D.C: US Government Printing Office; 2005. p. 1-19.

10. Nelson ME, Rejeski WJ, Blair SN, et al. Physical activity and public health in older adults: recommendation from the American College of Sports Medicine and the American Heart Association. Med Sci Sports Exerc. 2007; 39:1435-45. [PubMed: 17762378]

11. McGinnis B, Olson KL, Magid D, et al. Factors related to adherence to statin therapy. Ann Pharmacother. 2007; 41:1805-11. [PubMed: 17925498]

12. Perreault S, Blais L, Lamarre D, et al. Persistence and determinants of statin therapy among middle-aged patients for primary and secondary prevention. Br J Clin Pharmacol. 2005; 59:56473. [PubMed: 15842555]

13. Munger MA, Van Tassell BW, LaFleur J. Medication nonadherence: an unrecognized cardiovascular risk factor. MedGenMed. 2007; 9:58. [PubMed: 18092064]

14. Wang PS, Bohn RL, Knight E, Glynn RJ, Mogun H, Avorn J. Noncompliance with antihypertensive medications: the impact of depressive symptoms and psychosocial factors. J Gen Intern Med. 2002; 17:504-11. [PubMed: 12133140]

15. Clark PG, Nigg CR, Greene G, Riebe D, Saunders SD. The Study of Exercise and Nutrition in Older Rhode Islanders (SENIOR): translating theory into research. Health Educ Res. 2002; 17:552-61. [PubMed: 12408200]

16. Clark PG, Rossi JS, Greaney ML, et al. Intervening on exercise and nutrition in older adults: the Rhode Island SENIOR Project. J Aging Health. 2005; 17:753-78. [PubMed: 16377771]

17. Saunders SD, Greaney ML, Lees FD, Clark PG. Achieving recruitment goals through community partnerships: the SENIOR Project. Fam Community Health. 2003; 26:194-202. [PubMed: 12829941]

18. Mulligan JE, Greene GW, Caldwell M. Sources of folate and serum folate levels in older adults. J Am Diet Assoc. 2007; 107:495-9. [PubMed: 17324669]

19. United States Department of Agriculture. What we eat in America 1994-96, Continuing Survey of Food Intakes by Individuals (CSFII) 1994-1996. public use CD-ROM

20. Yaroch AL, Nebeling L, Thompson FE, et al. Baseline design elements and sample characteristics for seven sites participating in the Nutrition Working Group of the Behavior Change Consortium. J Nutr. 2008; 138:185S-192S. [PubMed: 18156423] 
21. Dipietro L, Caspersen CJ, Ostfeld AM, Nadel ER. A survey for assessing physical activity among older adults. Med Sci Sports Exerc. 1993; 25:628-42. [PubMed: 8492692]

22. Harada ND, Chiu V, King AC, Stewart AL. An evaluation of three self-report physical activity instruments for older adults. Med Sci Sports Exerc. 2001; 33:962-70. [PubMed: 11404662]

23. Young DR, Jee SH, Appel LJ. A comparison of the Yale Physical Activity Survey with other physical activity measures. Med Sci Sports Exerc. 2001; 33:955-61. [PubMed: 11404661]

24. Kruskall LJ, Campbell WW, Evans WJ. The Yale Physical Activity Survey for older adults: predictions in the energy expenditure due to physical activity. J Am Diet Assoc. 2004; 104:12517. [PubMed: 15281043]

25. Gidez LI, Miller GJ, Burstein M, Slagle S, Eder HA. Separation and quantitation of subclasses of human plasma high density lipoproteins by a simple precipitation procedure. J Lipid Res. 1982; 23:1206-23. [PubMed: 7175378]

26. Friedewald WT, Levy RI, Fredrickson DS. Estimation of the concentration of low-density lipoprotein cholesterol in plasma, without use of the preparative ultracentrifuge. Clin Chem. 1972; 18:499-502. [PubMed: 4337382]

27. Thompson FE, Subar AF, Smith AF, et al. Fruit and vegetable assessment: performance of 2 new short instruments and a food frequency questionnaire. J Am Diet Assoc. 2002; 102:1764-72. [PubMed: 12487538]

28. Boumendil EF. Descriptive study of lipid-modulating drug use in a French professional population. J Clin Epidemiol. 1994; 47:1163-71. [PubMed: 7722549]

29. Avorn J, Monette J, Lacour A, et al. Persistence of use of lipid-lowering medications: a crossnational study. Jama. 1998; 279:1458-62. [PubMed: 9600480]

30. Lytsy P, Westerling R. Patient expectations on lipid-lowering drugs. Patient Educ Couns. 2007; 67:143-50. [PubMed: 17433602] 
Table 1

Participant Descriptives

\begin{tabular}{|l|c|c|c|}
\hline & Whole Group & Taking Statins & Not Taking Statins \\
\hline $\mathrm{n}(\%)$ & & $37(32.2)$ & $78(67.8)$ \\
\hline Gender & & & \\
\hline Male, $\mathrm{n}(\%)$ & 33 & $11(33.3)$ & $22(66.7)$ \\
\hline Female, $\mathrm{n}(\%)$ & 82 & $26(31.7)$ & $56(68.3)$ \\
\hline Age, years & $75.2 \pm 6.8$ & $73.5 \pm 6.5$ & $75.8 \pm 6.8$ \\
\hline Smoking, $\%$ yes & 3.6 & 2.7 & 3.8 \\
\hline BMI, kg/m ${ }^{2}$ & $29.2 \pm 6.0$ & $29.4 \pm 6.8$ & $29.0 \pm 5.6$ \\
\hline Multivitamin, \% yes & 52.2 & 56.8 & 49.4 \\
\hline TC, mg/dL & $203.1 \pm 40.2$ & $187.0 \pm 37.8$ & $210.6 \pm 38 . *^{*}$ \\
\hline TAG, $\mathrm{mg} / \mathrm{dL}$ & $133.0 \pm 64.7$ & $132.2 \pm 57.1$ & $133.2 \pm 68.9$ \\
\hline HDL-C, mg/dL & $52.9 \pm 15.7$ & $54.8 \pm 18.6$ & $52.0 \pm 14.1$ \\
\hline LDL-C, mg/dL & $123.6 \pm 37.4$ & $105.7 \pm 31.6$ & $132.0 \pm 36.8$ \\
\hline YPAS Energy Expenditure ${ }^{1}, \mathrm{kcal}$ & $8,379 \pm 5,380$ & $7,973 \pm 3,860$ & $8,574 \pm 5.988$ \\
\hline YPAS Summary Score ${ }^{2}$ & $44.7 \pm 24.9$ & $45.4 \pm 22.9$ & $44.3 \pm 25.9$ \\
\hline
\end{tabular}

p $<0.05$

**

$\mathrm{p}<0.01$

1 YPAS Energy Expenditure n=106,

${ }^{2}$ YPAS Summary Score $n=110$ 
Table 2

Dietary Assessment of the Participants

\begin{tabular}{|c|c|c|c|}
\hline & Whole Group & Taking Statins & Not Taking statins \\
\hline Kcalories & $1,533 \pm 460$ & $1,458 \pm 460$ & $1,570 \pm 455$ \\
\hline Carbohydrates, $\%$ & $55.3 \pm 8.0$ & $54.5 \pm 8.3$ & $55.7 \pm 7.8$ \\
\hline Protein, $\%$ & $17.0 \pm 3.8$ & $17.3 \pm 3.9$ & $16.9 \pm 3.7$ \\
\hline Fat, $\%$ & $29.2 \pm 7.2$ & $29.7 \pm 8.3$ & $28.9 \pm 6.6$ \\
\hline Saturated fats, $\%$ & $9.4 \pm 3.1$ & $9.5 \pm 3.6$ & $9.4 \pm 2.9$ \\
\hline Polyunsaturated fats, $\%$ & $6.1 \pm 2.1$ & $6.3 \pm 2.1$ & $6.1 \pm 2.1$ \\
\hline Monounsaturated fats, $\%$ & $11.1 \pm 3.1$ & $11.3 \pm 3.6$ & $10.9 \pm 2.7$ \\
\hline Omega-3 fats, grams & $1.3 \pm 0.7$ & $1.3 \pm 0.8$ & $1.3 \pm 0.6$ \\
\hline Trans fat, grams & $3.5 \pm 2.0$ & $3.2 \pm 1.9$ & $3.6 \pm 2.0$ \\
\hline Cholesterol, mg & $218.0 \pm 143.9$ & $202.6 \pm 143.2$ & $226.1 \pm 143.7$ \\
\hline Fiber, grams & $17.0 \pm 6.8$ & $15.8 \pm 7.3$ & $17.7 \pm 6.5$ \\
\hline Fruit, servings & $2.6 \pm 1.3$ & $2.3 \pm 1.3$ & $2.7 \pm 1.3$ \\
\hline Vegetable, servings & $2.9 \pm 1.5$ & $2.5 \pm 1.6$ & $3.1 \pm 1.4^{*}$ \\
\hline F and V Total, servings & $5.5 \pm 2.2$ & $4.8 \pm 2.1$ & $5.9 \pm 2.2^{*}$ \\
\hline Vitamin A (retinol), mcg & $455.9 \pm 312.3$ & $441.5 \pm 378.0$ & $462.7 \pm 278.3$ \\
\hline Vitamin D, mcg & $4.6 \pm 2.9$ & $4.2 \pm 2.6$ & $4.9 \pm 3.1$ \\
\hline Vitamin E (aTOCeq), iu & $8.2 \pm 6.1$ & $7.0 \pm 5.0$ & $8.8 \pm 6.5$ \\
\hline Vitamin $\mathrm{K}$, mcg & $87.1 \pm 69.5$ & $68.7 \pm 48.7$ & $97.9 \pm 77.9^{*}$ \\
\hline Thiamin, mg & $116.1 \pm 56.4$ & $1.4 \pm 0.5$ & $1.5 \pm 0.5$ \\
\hline Riboflavin, mg & $1.8 \pm 0.7$ & $1.6 \pm 0.6$ & $1.8 \pm 0.7$ \\
\hline Niacin, mg & $20.1 \pm 7.5$ & $18.9 \pm 6.7$ & $20.6 \pm 7.8$ \\
\hline Vitamin $\mathrm{B}_{6}, \mathrm{mg}$ & $1.8 \pm 0.7$ & $1.7 \pm 0.8$ & $1.9 \pm 0.7$ \\
\hline Folate, mcg & $343.5 \pm 127.0$ & $318.7 \pm 130.8$ & $355.3 \pm 124.3$ \\
\hline Vitamin $B_{12}$, mcg & $7.7 \pm 16.0$ & $4.6 \pm 4.8$ & $9.4 \pm 19.1^{*}$ \\
\hline Vitamin C, mg & $116.1 \pm 56.4$ & $104.0 \pm 52.0$ & $123.1 \pm 58.6$ \\
\hline Sodium, mg & $2432.6 \pm 892.1$ & $2,358 \pm 954$ & $2,468 \pm 865$ \\
\hline Iron, mg & $14.1 \pm 7.0$ & $12.8 \pm 5.4$ & $14.8 \pm 7.5$ \\
\hline Calcium, mg & $708.6 \pm 335.9$ & $613.3 \pm 260.2$ & $753.7 \pm 356.8^{*}$ \\
\hline Magnesium, mg & $260.5 \pm 86.3$ & $239.7 \pm 79.4$ & $271.5 \pm 88.1$ \\
\hline Potassium, mg & $2652.5 \pm 850.4$ & $2407.5 \pm 898.8$ & $2779.4 \pm 806.9^{*}$ \\
\hline
\end{tabular}

$\mathrm{p}<0.05$ between groups 